\title{
Cancer-protective effects of inhaled corticosteroids in COPD are likely related to modification of epithelial activation
}

\author{
To the Editor:
}

The article published by RAYMAKERS et al. [1] presents a lung cancer-protective effect of inhaled corticosteroids (ICS) in patients with COPD. The authors suggest that this effect is due to "anti-inflammatory" properties of ICS on the airways. This may be partly true but, from what we have come to know of COPD pathogenesis, the situation is likely to be more nuanced and complicated than that.

We and others have published a number of papers on the characteristics of airway pathology in COPD patients [2-6]. We have found active epithelial-mesenchymal transition (EMT) as part of the gene reprogramming that occurs in epithelial basal cells, with subepithelial reticular basement membrane fragmentation and hypervascularity but underlying lamina propria fibrosis, hypovascularity and hypocellularity. In contrast to the airway wall, there is certainly innate immune activation in the airway lumen, and reactive oxygen species generated here may be a factor driving the EMT. However, we would suggest that it is the EMT and its related hypervascularity that is the driver of malignancy (so called type 3 EMT) in COPD. EMT also plays a role in progression and metastasis of lung cancers [7]. This may be a general paradigm for epithelial cancers, that incidentally make up 95\% of human malignancy.

Further, our investigations have also suggested that ICS can reverse some of the airway wall pathology in COPD, including airway EMT $[8,9]$. Therefore, it may well be that the cancer protection by ICS treatment demonstrated by RAYMAKers et al. [1] is not simply explained by an anti-inflammatory effect, but also perhaps mainly through an effect on EMT.

@ERSpublications

COPD is an independent risk factor for lung cancer. Studies have suggested that inhaled corticostroids have reduced the incidence of lung cancer in these patients. This effect is likely related to modification of epithelial-mesenchymal transition (EMT). http://bit.ly/2Km4VK4

Cite this article as: Soltani A, Mahmood MQ, Reid DW, et al. Cancer-protective effects of inhaled corticosteroids in COPD are likely related to modification of epithelial activation. Eur Respir J 2019; 54: 1901088 [https://doi.org/10.1183/13993003.01088-2019].

Amir Soltani ${ }^{1}$, Malik Q. Mahmood ${ }^{2}$, David W. Reid ${ }^{3}$ and E. Haydn Walters ${ }^{1}$

${ }^{1}$ Medical School, University of Tasmania, Hobart, Australia. ${ }^{2}$ School of Medicine, Deakin University, Waurn Ponds, Australia. ${ }^{3}$ QIMR-Berghofer Institute of Medical Research and University of Queensland, Brisbane, Australia.

Correspondence: E. Haydn Walters University of Tasmania, Discipline of Medicine, Private Bag 34, Hobart, 7001, Australia. E-mail: haydn.walters@utas.edu.au

Received: 03 June 2019 | Accepted: 17 June 2019

Conflict of interest: None declared.

\section{References}

1 Raymakers AJN, Sadatsafavi M, Sin DD, et al. Inhaled corticosteroids and the risk of lung cancer in COPD: a population-based cohort study. Eur Respir J 2019; 53: 1801257.

2 Soltani A, Muller HK, Sohal SS, et al. Distinctive characteristics of bronchial reticular basement membrane and vessel remodelling in chronic obstructive pulmonary disease (COPD) and in asthma: they are not the same disease. Histopathology 2012; 60: 964-970.

3 Soltani A, Reid DW, Sohal SS, et al. Basement membrane and vascular remodelling in smokers and chronic obstructive pulmonary disease: a cross-sectional study. Respir Res 2010; 11: 105.

4 Sohal SS, Reid D, Soltani A, et al. Reticular basement membrane fragmentation and potential epithelial mesenchymal transition is exaggerated in the airways of smokers with chronic obstructive pulmonary disease. Respirology 2010; 15: 930-938. 
5 Sohal SS, Reid D, Soltani A, et al. Evaluation of epithelial mesenchymal transition in patients with chronic obstructive pulmonary disease. Respir Res 2011; 12: 130.

6 Milara J, Peiró T, Serrano A, et al. Epithelial to mesenchymal transition is increased in patients with COPD and induced by cigarette smoke. Thorax 2013; 68: 410-420.

7 Mahmood MQ, Ward C, Muller HK, et al. Epithelial mesenchymal transition (EMT) and non-small cell lung cancer (NSCLC): a mutual association with airway disease. Med Oncol 2017; 34: 45.

8 Soltani A, Walters EH, Reid DW, et al. Inhaled corticosteroid normalizes some but not all airway vascular remodeling in COPD. Int J Chron Obstruct Pulmon Dis 2016; 11: 2359-2367.

9 Sohal SS, Soltani A, Reid D, et al. A randomized controlled trial of inhaled corticosteroids (ICS) on markers of epithelial-mesenchymal transition (EMT) in large airway samples in COPD: an exploratory proof of concept study. Int J Chron Obstruct Pulmon Dis 2014; 9: 533-542. 